\title{
Evaluation of total creatine kinase levels in a spectrum of neuro-psychiatric disorders in a tertiary neurosciences centre
}

\section{Anshu Gupta, Chhavi Gupta, Sarabjeet Khurana ${ }^{1}$}

Departments of Emergency Laboratory and ${ }^{1}$ Epidemiology, Institute of Human Behavior and Allied Sciences, New Delhi, India

\section{Address for the Correspondence:}

Dr. Anshu Gupta,

1408/13, Opposite Model School,

Civil Road, Rohtak - 124001

Haryana, India.

E-mail: dransh2002@yahoo.co.in

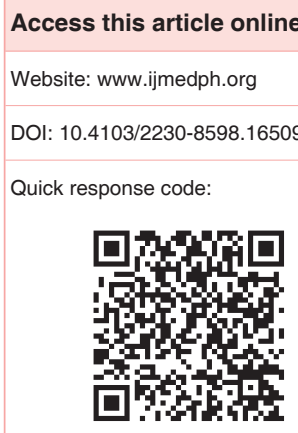

Introduction: To study usefulness of total creatine kinase (CK) as a screening tool in various neurological and psychiatric disorders in emergency setting of a tertiary care hospital. Materials and Methods: A 1-year retrospective study was conducted on 102 patients with complaints pertaining to neurological and psychiatric disorders in a tertiary neurosciences centre in a metropolitan city. Blood samples in plain vial were received in Emergency Laboratory and total CK levels were measured by automated analyzer and its correlation with various diseases was analyzed. Results: It was observed that CK activity was raised in various psychiatric conditions-acute transient psychotic disorder, alcohol dependence syndrome, delirium, psychosis, mental retardation, catatonia, bipolar affective disorder (BAD), depression and mania and also in neurological disorders-seizures, meningitis, myasthenia gravis (multiple sclerosis), Guillain-Barre syndrome, extra pyramidal syndrome, neuroleptic malignant syndrome and infarct. Conclusion: This study demonstrated that CK is a sensitive and an important screening parameter in diagnosis and monitoring of various neurological and psychiatric disorders in emergency setting. It is also helpful in identifying people at high risk for various neuro-psychiatric diseases.

Key words: Creatine kinase, emergency laboratory, neuropsychiatric disorders, screening

\section{INTRODUCTION}

Creatine kinase $(\mathrm{CK})$ is an enzyme with three isoforms BB/CK1, MB/CK2 and MM/CK3. The skeletal muscle contains primarily MM; cardiac muscle contains primarily MB and MM. Brain tissue, GI system and genitourinary tract contain primarily BB. Normally, total CK levels are virtually $100 \%$ MM isoenzyme. ${ }^{[1]}$ The CK level is raised in skeletal muscle dystrophies, inflammatory muscle diseases, exercise-induced rhabdomyolysis, snake bite poisoning, medication, frequent intramuscular injections, etc. Diagnosis of neurological and psychiatric disorders is based on clinical features, presence of risk factors, electromyography changes and biochemical findings. The diagnostic criteria used to classify psychiatric disorders were based on International statistical classification of Diseases-10, the more widely used counterpart of Diagnostic and Statistical manual of Mental Disorders-V. Total CK level is not only a useful screening tool but also significant for further monitoring of neurological and psychiatric disorders. ${ }^{[2,3]}$ Raised levels of total CK has been detected in the serum of patients with a variety of neurological and psychiatric disorders. ${ }^{[4,5]}$ We conducted the present study in the emergency laboratory of our hospital for assessing the utility of this test in neuropsychiatric disorders. The need for studying CK levels particularly in psychiatric disorders was felt to ascertain direct correlation between $\mathrm{CK}$ levels and psychiatry disorders rather than secondary changes associated with these disorders to cause its elevated levels.

\section{MATERIALS AND METHODS}

The subjects were selected from the patients who were admitted to the emergency department of a tertiary neurosciences hospital in a metropolitan city with various neurological and psychiatric complaints during the period from January 1, 2012 to December 31,2012. A total of 102 subjects were 
considered. Blood samples in plain vial from each patient within $6 \mathrm{~h}$ after admission were collected and received in emergency laboratory. The samples were then centrifuged and only non-hemolysed samples were analyzed within $1 \mathrm{~h}$ after collection. The following samples were rejected: Hemolysed samples, samples from patients with history of alcohol, and other drug intake, patients who had received multiple intramuscular injections and patients who have undergone strenuous exercise before sampling. In hemolysed samples, repeat samples were taken because enzymes and intermediates liberated from the red blood cells may affect the lag phase and the side reactions occurring in the assay system. ${ }^{[6]}$ Serum total CK test was carried out on the automated biochemistry system that used ENZOPAK NAC (kinetic) method which is based on modification of Szasz method ${ }^{[7]}$ and is according to International Federation of Clinical Chemistry. ${ }^{[8]}$ Serum total CK activity denotes CK-MM activity exclusively. The upper limit taken for normal CK was $140 \mathrm{U} / \mathrm{L}$ for women and $175 \mathrm{U} / \mathrm{L}$ for men.

\section{Statistical analysis}

It was performed using SPSS for windows 11.5 software program and Microsoft Excel 2007 Program with license no. 305147890.

\section{RESULTS}

The total study sample was composed of 102 cases, out of which $59(57.8 \%)$ were neurological cases and $43(42.2 \%)$ were psychiatric cases. The age distribution of the study sample is from 6 to 80 years of age with mean age 34.5 years as shown in contingency Table 1. The maximum number of study subjects was in the age group of $30-40$ years. There were 73 males and 29 females in the study group. The Reference range of total CK value taken was $38-175 \mathrm{U} / \mathrm{L}$ in males and $26-140 \mathrm{U} / \mathrm{L}$ in females. The total $\mathrm{CK}$ value in serum denotes exclusively CK-MM.

Among the neurological disorders, maximum number of cases was of seizures followed by meningitis, myasthenia gravis, Guillain-Barre Syndrome (GBS), extra pyramidal syndrome (EPS), neuroleptic malignant syndrome (NMS) and infarct. In the psychiatric disorders, the maximum number of cases were of acute transient psychotic disorder (ATPD) followed by alcohol dependence syndrome (ADS), psychosis, mental retardation (MR), delirium, catatonia, bipolar affective disorder (BAD), depression and mania [Pie Charts 1 and 2].

In neurological disorders, CK level was found to be significantly increased in maximum cases of seizures $(67.7 \%)$ followed by meningitis $(10.1 \%)$. In most of the neurological cases $(84.7 \%)$, total $\mathrm{CK}$ value was significantly increased up to $1500 \mathrm{U} / \mathrm{L}$ followed by $10.2 \%$ cases of seizures and meningitis with CK values between 1500 and $3000 \mathrm{U} / \mathrm{L}$ and few cases $(5.1 \%$ ) of seizures and myasthenia gravis with total CK value exceeding $3000 \mathrm{U} / \mathrm{L}$, irrespective of gender.

In psychiatric conditions, maximum number of cases $(83.7 \%)$ showed total CK values increased up to $1500 \mathrm{U} / \mathrm{L}$ followed by $9.3 \%$ cases of psychosis, ADS, MR and catatonia with total $\mathrm{CK}$ values between 1500 and $3000 \mathrm{U} / \mathrm{L}$ and $6.9 \%$ cases of alcohol dependence syndrome and catatonia had total CK values above $3000 \mathrm{U} / 1$, irrespective of gender [Tables 2 and 3].

\section{DISCUSSION}

Creatine kinase, also known as creatine phosphokinase is an enzyme expressed by various tissues and cell types that catalyses the reversible phosphorylation of creatine by adenosine triphosphate..$^{[9]}$ CK activity is greatest in striated muscle and heart tissue and then in other tissues such as brain, the GIT and the urinary bladder. CK is a dimer composed of two subunits-B and M. Three different forms of subunits exist-BB, MB and MM. CK-MM is found mainly

\begin{tabular}{lcc}
\hline \multicolumn{3}{l}{ Table 1: Age distribution of the study samples } \\
\hline Age group (in years) & Number of patients & Percentage \\
\hline $0-10$ & 04 & 4.08 \\
$10-20$ & 19 & 19.38 \\
$20-30$ & 17 & 17.34 \\
$30-40$ & 38 & 38.76 \\
$40-50$ & 06 & 6.12 \\
$50-60$ & 08 & 8.16 \\
$60-70$ & 07 & 7.14 \\
$70-80$ & 03 & 3.06 \\
Total & 102 & \\
\hline
\end{tabular}

\begin{tabular}{|c|c|c|c|c|}
\hline Total CK value (U/L) & $>\mathrm{RV}-1500$ & $1500-3000$ & $3000-4500$ & Total \\
\hline Seizure & 33 & 5 & 2 & 40 \\
\hline Meningitis & 5 & 1 & 0 & 6 \\
\hline Myasthenia gravis & 3 & 0 & 1 & 4 \\
\hline GBS & 3 & 0 & 0 & 3 \\
\hline EPS & 2 & 0 & 0 & 2 \\
\hline NMS & 2 & 0 & 0 & 2 \\
\hline Infarct & 2 & 0 & 0 & 2 \\
\hline Total & 50 & 6 & 3 & 59 \\
\hline Percentage & 84.7 & 10.2 & 5.1 & \\
\hline
\end{tabular}

GBS = Guillain-Barre syndrome, EPS = Extra pyramidal syndrome, NMS = Neuroleptic malignant syndrome, $C K=$ Creatine kinase

\begin{tabular}{|c|c|c|c|c|}
\hline Total CK value (U/L) & $>\mathrm{RV}-1500$ & $1500-3000$ & $3000-4500$ & Total \\
\hline ATPD & 8 & 0 & 0 & 8 \\
\hline Psychosis & 6 & 1 & 0 & 7 \\
\hline ADS & 5 & 1 & 2 & 8 \\
\hline Delirium & 5 & 0 & 0 & 5 \\
\hline MR & 4 & 1 & 0 & 5 \\
\hline Catatonia & 2 & 1 & 1 & 4 \\
\hline BPAD & 2 & 0 & 0 & 2 \\
\hline Depression & 2 & 0 & 0 & 2 \\
\hline Mania & 2 & 0 & 0 & 2 \\
\hline Total & 36 & 4 & 3 & 43 \\
\hline Percentage & 83.7 & 9.3 & 6.9 & \\
\hline
\end{tabular}

$\mathrm{RV}=$ Reference value, $\mathrm{ATPD}=$ Acute transient psychotic disorder, $\mathrm{ADS}=$ Alcohol dependence syndrome, $\mathrm{MR}=$ Mental retardation, $\mathrm{BAD}=$ Bipolar affective disorder, $\mathrm{CK}=$ Creatine kinase 


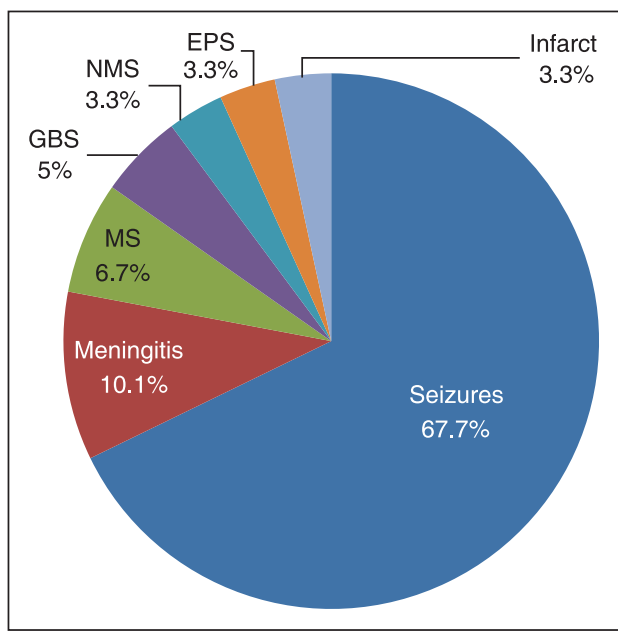

Pie Chart 1: Distribution of study subjects with neurological disorders

in skeletal muscle and then in heart, CK-MB is demonstrable in the heart but is also found in the skeletal muscle and urinary bladder. CK-BB is mainly found in the brain, GIT and urinary bladder. More than $20 \%$ of the total CK activity is present as CK-MB and nearly $80 \%$ as CK-MM. Thus, in healthy individuals, the total CK activity in the serum consists almost exclusively of CK-MM. ${ }^{[10]} \mathrm{CK}$ isoenzymes can be quantitated by electrophoretic or immunologic methods. Because CK exists in relatively few organs, elevated serum total CK level estimation is used as a specific index of injury to muscle and myocardium. ${ }^{[1]}$

Raised levels have been detected in the serum of patients with a variety of neurological conditions such as seizures, myasthenia gravis (multiple sclerosis), GBS, EPS, etc. ${ }^{[4,5]}$ In our study, total CK level was found to be significantly increased in maximum cases of seizures $(67.7 \%)$ followed by meningitis $(10.1 \%)$. CK level was raised in few cases of GBS, EPS, NMS, Myasthenia gravis and Infarct (4-7\%). In previous study by Libman et al., they measured serum CK in seizure versus syncope cases in the emergency department and found that mean serum CK level was higher in seizure group (231.1 \pm 34.8$)$ as compared to syncope cases $(70.5 \pm 5.6)$. They concluded that CK level is highly specific test for diagnosing generalized seizures in the emergency department. ${ }^{[12]}$ Petramfar et al., investigated CK concentration to distinguish between generalized tonic-clonic seizures (GTCS) from either psychogenic non epileptic seizures (PNES) or vasovagal syncope (VVS). It was observed that CK concentration was increased in $75 \%$ of patients with GTCS as compared to $15 \%$, $13.6 \%$ and $15 \%$ of patients with PNES, VVS and control group respectively. Thus, CK concentration is also a useful and relatively accurate parameter to assist in the differentiation of epileptic seizures from either VVS or PNES. ${ }^{[13]}$ Sharma and Nand evaluated $\mathrm{CK}$ enzyme in pyogenic and tuberculous meningitis and reported that $\mathrm{CK}$ levels were significantly elevated in cases of meningitis. ${ }^{[14]}$

Neuroleptic malignant syndrome is characterized by clinical symptoms that include hyperthermia, severe muscle rigidity, autonomic dysfunction and altered mental state. Nisijima in a study concluded that an elevated CK level is common in the early stage

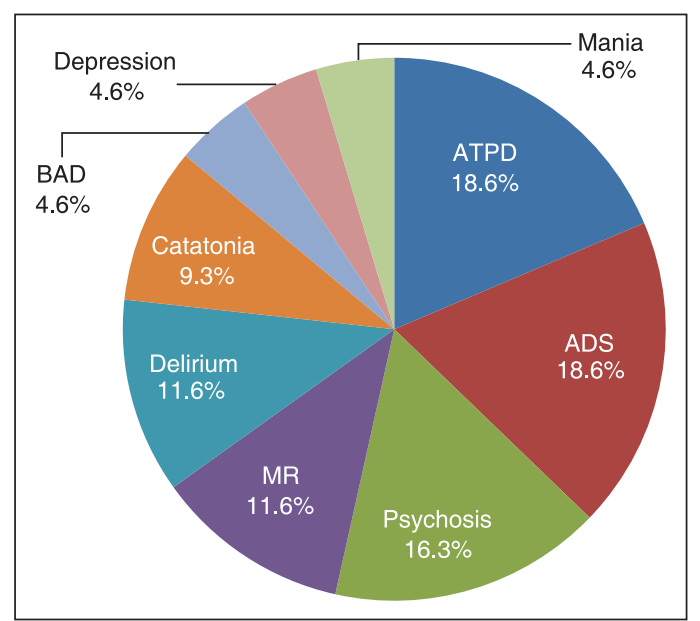

Pie Chart 2: Distribution of study subjects with psychiatric disorders

of NMS and corresponds temporally with the onset of muscle rigidity ${ }^{[15]}$ while another study by O'Dwyer and Sheppard suggested that elevation of CK in NMS is a non-specific finding and its role in the diagnosis of the syndrome remains controversial. ${ }^{[16]}$ In EPS and Parkinson disease, mechanism mediated by hypothalamic dopamine and by autonomic nervous system may also be contributing to the elevation of serum CK as proved by Takubo et al..$^{[1]}$

Some studies observed that serum CK-MB and total CK levels were elevated above the cutoff value in patients with stroke/infarction. These enzyme levels gradually increased within the first 3 days and declined afterwards. ${ }^{[18]}$

The neuropathies cause denervation of muscle fibers and the ongoing entrophic changes to the muscle fiber membrane that result in leakage of CK. Another possibility would be elevation of CK secondary to frequent cramping, a common symptom in acute neurogenic diseases such as GBS. ${ }^{[19]}$ Similarly, in myasthenia gravis, there is possible leakage of enzyme and metabolite from the myasthenic muscle due to a membrane defect and there may be a myopathic component also leading to increase in CK level. [20]

The association between psychiatric disorders and raised total CK levels remain argumentative. In our study, raised CK levels were found in maximum cases of ATPD and ADS ( $8 \%$ each) followed by psychosis $(6 \%)$, delirium tremens $(5 \%)$ and MR (4\%). Few cases of depression, mania and BAD (2\% each) had raised CK levels in the serum. CK was found to be elevated particularly in those patients who had history of intake of neuroleptic drugs.

Previous studies in schizophrenic patients indicated elevation of serum CK levels, mainly of MM isoenzyme but this test did not appear to be diagnostic in schizophrenia. Histological changes such as ' $Z$ ' band spreading had been reported in skeletal muscles which were interpreted as secondary consequences of an abnormality in nervous system factors from acute schizophrenics. ${ }^{[21]}$

Some theories relate elevated CK level to psychomotor agitation and medication in acute psychosis. Hermesh et al. showed absence of 
myoglobinuria in acute psychotic patients with marked elevation in serum CK level concluding that CKemia may be due to an unknown, nontraumatic, pathophysiological mechanism. ${ }^{[2]}$ There have been few studies focusing on $\mathrm{CK}$ elevations in chronic psychiatric conditions. One of the studies by Terao (2012) suggested that a few chronic psychiatric inpatients had CK elevation but patients engaged in more physical activity or receiving higher neuroleptic drugs are at greater risk of developing CK elevations. However, most of these increases are benign and need no treatment. ${ }^{[23]}$ There is also evidence of neuromuscular dysfunction in psychotic patients. Increase in permeability of the muscle membrane due to some defect in the neurotropic regulation of muscle physiology or an independent expression of muscle abnormalties may lead to higher serum CK activity. ${ }^{[2]}$

Creatine kinase level was maximally increased in alcohol abuse in the range of 3000-4500U/L in our study which conforms to the findings of Osborn et al. He evaluated CK and CK-MB in alcohol abuse subjects with recent heavy consumption and concluded that elevated CK levels are common in heavy alcohol use patients without evidence of myocardial ischemia. ${ }^{[25]}$ Contrary to our findings, Segal et al. studied serum CK levels in alcohol syndromes and found low normal CK activity for alcohol dependence, higher for alcohol withdrawal and the highest for delirum tremens. These findings may be related to enhanced dopamine activity in alcohol dependence as increased dopamine activity inhibits CK and conversely, to a significant decrease in dopamine activity during withdrawal syndromes. ${ }^{[26]}$

In catatonia, increased serum creatine phosphokinase may be related to occurrence of dyskinetic movements. ${ }^{[27]}$

Segal et al. also studied serum CK levels in various forms of depression. They reported 'low normal' CK levels, especially in psychotic depressions. ${ }^{[28]}$

Previous studies reported elevated CK levels in MR and depressive, maniac and euthymic phases of bipolar disorder. ${ }^{[29]}$

There are certain limitations to our study such as inadequate documentation available due to retrospective study undertaken and small sample size.

\section{CONCLUSION}

Our present study confirms serum total CK level as a sensitive indicator in neurological as well as psychiatric disorders as it is found to be raised in all cases but requires detailed diagnostic work up, including electrophysiological, histopathological and genetic studies to reach at a final conclusion. CK activity was found to be maximally increased in the range of 3000-4500U/L in seizures and meningitis among neurological disorders and in alcohol abuse among psychiatric disorders. It seems likely that the predominant source of increased CK activity in serum is the skeletal muscles in neuropsychiatric disorders. Nevertheless, the origin of total CK elevations in these patients remains to be elucidated further. In psychiatric disorders, there could be primary changes such as neuromuscular defect, defect in nervous system secretory factors or there could be secondary changes such as alcohol or other drug intake or increased physical activity that lead to elevated CK level activity. No primary specific factor found to be causing elevation of $\mathrm{CK}$ level but as we measured primarily CK-MM, we can conclude that all these disorders lead to changes in muscle ultimately. Comprehensive studies are needed to understand the differences in serum kinase activity in various conditions by analyzing gender and age differences, isozyme patterns of $\mathrm{CK}$ and alterations in central and peripheral dopamine indices.

Despite all the limitations, a simple and rapid evaluation of serum CK activity still provide a reliable supplementary screening tool in various neuropsychiatric disorders particularly in emergency settings and helps in taking immediate treatment decisions.

\section{REFERENCES}

1. Nanji AA. Serum creatine kinase isoenzymes: A review. Muscle Nerve 1983;6:83-90.

2. Bell RD, Rosenberg RN, Ting R, Mukherjee A, Stone MJ, Willerson JT. Creatine kinase BB isoenzyme levels by radioimmunoassay in patients with neurological disease. Ann Neurol 1978;3:52-9.

3. Phillips JP, Jones HM, Hitchcock R, Adama N, Thompson RJ. Radioimmunoassay of serum creatine kinase BB as index of brain damage after head injury. Br Med J 1980;281:777-9.

4. Thompson RJ, Graham JG, McQueen IN, Kynoch PA, Brown KW. Radioimmunoassay of brain-type creatine kinase-BB isoenzyme in human tissues and in serum of patients with neurological disorders. J Neurol Sci 1980;47:241-54.

5. Tsung $\mathrm{SH}$. Several conditions causing elevation of serum CK-MB and CK-BB. Am J Clin Pathol 1981;75:711-5.

6. Panteghini M, Bais R, Solinge WW. Enzymes. In: Burtis CA, Ashwood ER, Bruns DE, editors. Tietz textbook of clinical chemistry and molecular diagnostics. Elsevier, Missouri: $4^{\text {th }}$ ed, 2006. p. 597-643.

7. Szasz G. Laboratory measurement of creatine kinase activity. In: Tietz NW, Weinstock A, Rogerson DO, editors. Proceedings of the $2^{\text {nd }}$ International Symposium in Clinical Enzymology. Washington, DC: American Association of Clinical Chemistry; 1976. p. 143.

8. Horder M, Elser RC, Gerhardt W, Mathieu M, Sampson EJ. Intrnational Federation of clinical chemistry (IFCC): Scientific Division Committee on enzymes. J Auto Chem 1990;12:22-40.

9. Goldblatt $\mathrm{H}$. The effect of high salt intake on the blood pressure of rabbits. Lab Invest 1969;21:126-8.

10. Babu GG, Walker JM, Yellon DM, Hausenloy DJ. Peri-procedural myocardial injury during percutaneous coronary intervention: An important target for cardioprotection. Eur Heart J 2011;32:23-31.

11. Fischbach F. Creatine phosphokinase. In: Rader I, editors. A Manual of Laboratory \& Diagnostic Tests. $6^{\text {th }}$ ed. Philadelphia: Lippincott; 2000. p. 441-4.

12. Libman MD, Potvin L, Coupal L, Grover SA. Seizure vs. syncope: Measuring serum creatine kinase in the emergency department. J Gen Intern Med 1991;6:408-12.

13. Petramfar P, Yaghoobi E, Nemati R, Asadi-Pooya AA. Serum creatine phosphokinase is helpful in distinguishing generalized tonic-clonic seizures from psychogenic nonepileptic seizures and vasovagal syncope. Epilepsy Behav 2009;15:330-2.

14. Sharma M, Nand N. Evaluation of enzymes in pyogenic and tuberculous meningitis. J Assoc Physicians India 2006;54:118-21.

15. Nisijima K. Elevated creatine kinase does not necessarily correspond temporally with onset of muscle rigidity in neuroleptic malignant syndrome: A report of two cases. Neuropsychiatr Dis Treat 2012;8:615-8.

16. O'Dwyer AM, Sheppard NP. The role of creatine kinase in the diagnosis of neuroleptic malignant syndrome. Psychol Med 1993;23:323-6.

17. Takubo H, Shimoda-Matsubayashi S, Mizuno Y. Serum creatine kinase is elevated in patients with Parkinson's disease: A case controlled study. Parkinsonism Relat Disord 2003;9 Suppl 1:S43-6. 
18. Ay $\mathrm{H}$, Arsava EM, Saribas O. Creatine kinase-MB elevation after stroke is not cardiac in origin: Comparison with troponin $T$ levels. Stroke 2002;33:286-9.

19. Satoh J, Okada K, Kishi T, Nagayama S, Kuroda Y. Cramping pain and prolonged elevation of serum creatine kinase levels in a patient with Guillain-Barré syndrome following Campylobacter jejuni enteritis. Eur J Neurol 2000;7:107-9.

20. Kolins J, Gilroy J. Serum enzyme levels in patients with myasthenia gravis afte aerobic and ischaemic exercise. J Neurol Neurosurg Psychiatry 1972;35:34-40.

21. Kondaiah P, Murthy KK, Reddi OS. Plasma creatine phosphokinase in schizophrenia. Indian J Psychiatry 1981;23:351-2.

22. Hermesh H, Manor I, Shiloh R, Weizman R, Munitz H. Absence of myoglobinuria in acute psychotic patients with marked elevation in serum creatine phosphokinase level. Eur Neuropsychopharmacol 2001;11:111-5.

23. Terao T, Matsuda S, Kojima H, Okuno K, Hori H, Kaku A, Ueda N, Etoh A. Incidence and risk factors of benign creatine phosphokinase elevations in chronic psychiatric patients. Neuropsychobiology 1999;39:173-80.

24. Meltzer HY, Ross-Stanton J, Schlessinger S. Mean serum creatine kinase activity in patients with functional psychoses. Arch Gen Psychiatry 1980;37:650-5.

25. Osborn LA, Rossum A, Standefer J, Jackson J, Skipper B, Beeson C, et al. Evaluation of CK and CK-MB in alcohol abuse subjects with recent heavy consumption. Cardiology 1995;86:130-4.

26. Segal M, Avital A, Rusakov A, Sandbank S, Weizman A. Serum creatine kinase activity differentiates alcohol syndromes of dependence, withdrawal and delirium tremens. Eur Neuropsychopharmacol 2009;19:92-6.

27. Northoff G, Wenke J, Pflug B. Increase of serum creatine phosphokinase in catatonia: An investigation in 32 acute catatonic patients. Psychol Med 1996;26:547-53.

28. Segal M, Avital A, Drobot M, Lukanin A, Derevenski A, Sandbank S, et al. Serum CK level in unmedicated non psychotic, psychotic, bipolar and schizoaffective depressed patients. Eur Neuropsychopharmacol J 2007; 17:194-8.

29. Feier G, Valvassori SS, Rezin GT, Búrigo M, Streck EL, Kapczinski F, et al. Creatine kinase levels in patients with bipolar disorder: Depressive, manic, and euthymic phases. Rev Bras Psiquiatr 2011;33:171-5.

How to cite this article: Gupta A, Gupta C, Khurana S. Evaluation of total creatine kinase levels in a spectrum of neuro-psychiatric disorders in a tertiary neurosciences centre. Int J Med Public Health 2015;5:362-6.

Source of Support: Nil, Conflicts of Interest: None declared. 